\title{
Comorbidity between headache and epilepsy in a pediatric headache center
}

\author{
Irene Toldo $\cdot$ Egle Perissinotto $\cdot$ Francesca Menegazzo $\cdot$ Clementina Boniver \\ Stefano Sartori $\cdot$ Leonardo Salviati $\cdot$ Maurizio Clementi $\cdot$ Pasquale Montagna • \\ Pier Antonio Battistella
}

Received: 16 October 2009/Accepted: 12 January 2010/Published online: 29 January 2010

(C) Springer-Verlag 2010

\begin{abstract}
The purpose of this study was to analyse the comorbidity between headache and epilepsy in a large series of children with headache $(1,795)$. Fifty-six cases (3.1\%) suffered from idiopathic headache and idiopathic or cryptogenic epilepsy or unprovoked seizures. There was a strong association between migraine and epilepsy: in migraineurs (46/56) the risk of epilepsy was 3.2 times higher when compared with tension-type headache, without significant difference between migraine with and without aura $(P=0.89)$; children with epilepsy had a 4.5 -fold increased risk of developing migraine than tension-type headache. In cases with comorbidity, focal epilepsies prevailed (43/56, $76.8 \%)$. Migraineurs affected by focal epilepsies (36/56) had a three times higher risk of having a cryptogenic epilepsy $(27 / 36,75 \%)$ than an idiopathic epilepsy $(9 / 36,25 \%)$ $(P=0.003)$. In migraine with aura, epilepsy preceded migraine in $71 \%$ of cases. Photosensitivity $(7 / 56,12.5 \%)$ and positive family history for epilepsy $(22 / 56,39 \%)$ were frequent in cases with comorbidity.
\end{abstract}

Keywords Headache $\cdot$ Migraine $\cdot$ Epilepsy · Comorbidity $\cdot$ Children

I. Toldo $(\bowtie) \cdot$ F. Menegazzo $\cdot$ C. Boniver $\cdot$ S. Sartori ·

L. Salviati - M. Clementi · P. A. Battistella

Department of Pediatrics, University of Padova, Padua, Italy

e-mail: irene.toldo@unipd.it

E. Perissinotto

Department of Preventive Medicine and Public Health, University of Padova, Padua, Italy

P. Montagna

Department of Neurological Sciences, University of Bologna, Bologna, Italy

\section{Introduction}

Migraine and epilepsy are both common neurological disorders, although migraine is more frequent, and can occur as comorbid conditions [1-6]. An association between migraine and epilepsy has been demonstrated in several studies, mainly based on adults affected by epilepsy [1, 5, 6]. In children an association between migraine and some epilepsy syndromes has been reported, but data are conflicting and studies have been limited by the small numbers of patients and to the lack of clearly stated diagnostic criteria of childhood migraine [7].

The growing knowledge of the genetic bases of familial hemiplegic migraine (FHM) [8] and the findings of epileptic seizures occurring independent of the migraine attacks in FHM families [9] have stimulated the interest on the comorbidity between migraine and epilepsy.

The purpose of this study was to analyse the comorbidity between headache and epilepsy in a large series of children and to find out possible correlations among the type of headache, the type of epilepsy of probands and the family history for headache and epilepsy.

\section{Methods}

This is a retrospective case-control study of 1,795 patients under 18 years of age consecutively diagnosed as affected by headache at the pediatric Headache Center of the Department of Pediatrics of Padua Hospital between 1995 and 2008. We reviewed the medical records of all 1,795 patients, which contain pre-established questions concerning family and personal history of headache, and past medical history, which includes a section on seizures and epilepsy. From the total study population, we identified 56 
patients with a diagnosis of idiopathic headache associated with idiopathic or cryptogenic epilepsy or unprovoked seizure. Patients with secondary headache or symptomatic epilepsy were excluded from the study. The study protocol and consent/assent forms were approved by our Institutional Review Board.

All 56 children included in the research and their parents were informed about the complete study protocol by letter and subsequently were contacted by telephone. They were asked to participate in a structured interview conducted in 2008 by a board certified child neurologist, regarding headache, epilepsy, and "periodic syndromes" in their child and other family members. Particular attention was devoted to the clinical features of headache and epilepsy. All 56 patients underwent brain neuroimaging (MRI or CT) and EEG with electrode placement according to the International 10-20 System. We reviewed all clinical, electrophysiological, and neuroimaging data. Forty-one patients were clinically reevaluated at a control visit in the second semester of 2008, while the other 15 patients participated only to the telephone clinical interview for follow-up.

The diagnosis of headache was based on the International Classification of Headache Disorders (ICHD-II) criteria [10]. The diagnosis of epilepsy was based on clinical and EEG data and classified according to the International Classification of Epilepsy and Epilepsy Syndromes [11, 12]. Focal cryptogenic epilepsies were further classified on the basis of the origin of epileptogenic focus (frontal, temporal, parietal, or occipital lobe).

All the data were entered into a database and analyzed with SAS statistical package (9.2 version). Data were analyzed by a chi-square statistic technique and, in case of low sample, by the exact Fischer test. We calculated measures of association between variables expressed by the odds ratio (OR), for which the confidence interval (CI) to 95\% was determined; for each test the level of significance was set at 0.05 .

\section{Results}

Information concerning headache and seizures was collected from 1,795 headache patients under 18 years of age consecutively diagnosed at the Headache Center of the Department of Pediatrics of the University of Padua between 1995 and 2008 (Table 1). Fifty-six patients $(N=56 / 1,795,3.1 \%)$ were identified as having comorbidity between primary headache and idiopathic or cryptogenic epilepsy or unprovoked seizures. There were 20 males $(35.7 \%)$ and 36 females $(64.3 \%)$ with a maleto-female ratio of 1:1.8. The average age at headache onset was 8.6 years (range 3-17 years, SD 4.3 years),
Table 1 Clinical characteristics of our population

\begin{tabular}{lll}
\hline & $N$ & $\%$ \\
\hline Sex & & \\
Male & 835 & 46.5 \\
Female & 960 & \\
Total & 1,795 & \\
Migraine & 957 & 53.3 \\
MO & 836 & \\
MA & 121 & \\
Male to female ratio (M/F) & $1 / 1.1$ & \\
Tension type & 686 & 38.2 \\
ETTH & 621 & \\
CTTH & 65 & \\
Male to female ratio (M/F) & $1 / 1.3$ & \\
Other headache types & 152 & 8.5 \\
Other primary headaches & 132 & \\
Headache attributed to psychiatric disorder & 14 & \\
Secondary headaches & 6 & 0.3 \\
Seizures & 68 & \\
Cases selected & \\
\hline
\end{tabular}

a Inclusion criteria: idiopathic or cryptogenic epilepsy or unprovoked seizures

$M O$ migraine without aura, $M A$ migraine with aura, ETTH episodic tension type headache, $C T T H$ chronic tension type headache

while the average age at epilepsy onset was 7.8 years (range 1-24 years, SD 4.0 years). In 25 subjects (44\%) epilepsy preceded headache, in 16 subjects $(28.6 \%)$ headache started in the same year of epilepsy, and in 15 cases (27.4\%) headache preceded epilepsy. In patients affected by migraine with aura (MA), epilepsy preceded migraine in $71 \%$ of the cases.

\section{Headache}

A total of 46 out of 56 subjects (82.1\%) had migraine, with a second level of diagnosis of migraine without aura (MO) in $69.6 \%(N=39)$ and of MA in $12.5 \%(N=7)$ of cases while 10 subjects $(17.9 \%)$ had episodic tension type headache (ETTH); no other types of headache were reported.

Among the 1,795 patients, comparing the distribution of headache type in cases, respectively, with and without epilepsy, we found that the prevalence of migraine in cases with epilepsy $(N=46 / 56,82.1 \%)$ was significantly higher compared to cases without epilepsy $(N=911 / 1739,52 \%)$ $(P=0.0006)$. The risk of migraine in patients with epilepsy was on average 4.5 times higher compared to the risk of ETTH, without a significant difference between $\mathrm{MO}$ and MA (respectively, 4.6 and 5.0, $P=0.89$ ). The ratio between MA and MO was 1:5.6 $(N=7 / 39)$. 
Nineteen out of 56 cases $(34 \%)$ had post-ictal headache, fulfilling the ICHD-II criteria (7.6.2) [10]. Many patients $(N=16 / 19,84 \%)$ with post-ictal headache had migraine attacks independent of epileptic seizures; however, migraineurs did not have an increased risk of post-ictal headache compared to patients with ETTH $(P=0.58)$. Among the seven patients with MA, nobody experienced episodes of migralepsy.

\section{Epilepsy}

Epilepsy was significantly more frequent in migraineurs $(N=46 / 957,4.8 \%)$ than in patients with ETTH $(N=$ $10 / 686,1.5 \%)(P=0.0001)$. The risk of epilepsy $(\mathrm{OR})$ in migraineurs was 3.2 times higher (CI of $95 \%=1.7-6.2$ ) compared to patients with ETTH and did not differ significantly between $\mathrm{MO}$ and MA cases. The most frequent type of epilepsy was focal epilepsy $(N=43 / 56,76.8 \%)$ among which focal cryptogenic epilepsies prevailed $(N=32 / 56,57.1 \%$ ) (Table 2). The distribution of epilepsy type (focal idiopathic, focal cryptogenic, generalized, and isolated seizures) did not differ between migraine and ETTH $(P=0.77)$, neither between $\mathrm{MO}$ and MA $(P=0.9)$.

However, among migraineurs with focal epilepsy, the risk of having a cryptogenic epilepsy was about three times higher than an idiopathic epilepsy $(P=0.003)$. In the subjects affected by migraine and focal cryptogenic epilepsies $(N=27)$, those originating in temporal and occipital lobes $(N=16)$ prevailed on frontal epilepsies $(N=6)(P=0.06)$.

On interictal EEG during intermittent photic stimulation (IPS), seven $(N=7 / 56,12.5 \%)$ patients had a photoparoxysmal response (PPR), two of whom also had a photoconvulsive response. PPR was characterized by generalized discharges in most of the cases $(N=6 / 7,85.7 \%)$, and only one patient had bilateral discharges on occipital regions during IPS.

An ictal EEG recording was performed in 14 (25\%) patients, and half of them (7/14) had focal seizures recorded. In particular five cases presented focal seizures with parossistic activity on left temporal (1/5), left central (1/5) with also an electrical status epilepticus during slow sleep on EEG, frontal bilateral (1/5), right frontal (2/5) and two cases had a focal epileptic status (1 right temporo-occipital, 1 right frontotemporal). In the remaining seven cases, two had palpebral myoclonia, four had absences (one of whom absence status), and one had subtle focal seizures during ictal EEG.

In other six cases a post-ictal EEG recording was obtained.

Family history of migraine and epilepsy in probands

A positive family history of headache and migraine was found respectively, in $73.2 \%(N=41 / 56)$ and in $66 \%$ $(N=37 / 56)$ of our cases. In the latter, $81 \%(N=30 / 37)$ had at least one-first-degree relative affected by migraine, mainly on maternal than on paternal lineage (respectively, $N=$ $23 / 30,77 \%$ vs. $N=8 / 30,27 \%$ ). All first-degree familial MA cases $(N=3 / 56)$ were exclusively of maternal origin. There was not any statistical significant correlation between the positive family history of migraine and the type of headache or epilepsy of the probands. A positive family history of non-symptomatic epilepsy was found in $39 \%$ $(N=22 / 56)$ of probands and $12.5 \%(N=7 / 56)$ of probands had at least one-first-degree relative affected by epilepsy.

\section{Discussion}

In previous studies, the prevalence of migraine in epileptic populations was estimated to be $8-24 \%[1,2,5,6]$ and the

Table 2 Distribution of headache diagnosis on the basis of type of epilepsy

\begin{tabular}{|c|c|c|c|}
\hline & $\begin{array}{l}\text { Total } \\
N=56(\%)\end{array}$ & $\begin{array}{l}\text { Migraine } \\
N=46(\%)\end{array}$ & $\begin{array}{l}\text { Episodic tension } \\
\text { type headache } \\
N=10(\%)\end{array}$ \\
\hline Focal cryptogenic epilepsy & $32(57.1)$ & $27 / 32(84)$ & $5 / 32(16)$ \\
\hline Site determined & 27 & $22 / 27$ & $5 / 5$ \\
\hline Frontal & 9 & $6 / 9$ & $3 / 9$ \\
\hline Temporo-occipital & 18 & $16 / 18$ & $2 / 18$ \\
\hline Site not determined & 5 & $5 / 27$ & $0 / 5$ \\
\hline Focal idiopathic epilepsy & $11(19.7)$ & $9 / 11(82)$ & 2/11 (18) \\
\hline Rolandic & 9 & $7 / 9$ & $2 / 9$ \\
\hline Occipital & 2 & $2 / 2$ & $0 / 2$ \\
\hline Idiopathic generalized epilepsy & $10(17.9)$ & $8 / 10(80)$ & $2 / 10(20)$ \\
\hline Isolated unprovoked seizures & $3(5.3)$ & $2 / 3(67)$ & $1 / 3(33)$ \\
\hline
\end{tabular}


risk of migraine was 2.4 times higher among adults with epilepsy than in those without $[1,5,6]$. The prevalence of migraine in children with partial epilepsy was $21 \%$ in benign rolandic epilepsy and $17 \%$ in cryptogenic/symptomatic epilepsy [7].

In the last 20 years, few studies have been performed concerning the comorbidity between headache and epilepsy in children [3, 4, 7, 13-17] and the majority of them are based on series of subjects affected by epilepsy [3, 4, 7, $13,14,16,17]$. Due to the different methodologies used they are quite difficult to compare. To our knowledge, this is the first study that evaluated the occurrence of epilepsy in a large series of children affected by headache, using the recent ICHD-II criteria [10] and with a longitudinal follow-up.

We found a statistically significant association between migraine and epilepsy in our series. In fact the prevalence of migraine in cases with epilepsy $(82.1 \%)$ was significantly higher than in those without epilepsy (52\%) and children with epilepsy had a 4.5-fold increased risk of developing migraine than tension-type headache.

In a prospective study on 50 children with epilepsy, $46 \%$ had headache and 10 out of the $23(43.5 \%)$ headache sufferers had migraine [13]. Most patients with headache were older than 10 years $(54.5 \%)$ and had idiopathic epilepsy (65.2\%) [13]. Different from this study, we found that the male-to-female ratio was significantly skewed in favor to females $(P=0.03)$, most patients with headache and epilepsy were younger than 10 years old $(60 \%)$, and suffered from cryptogenic epilepsy (60\%) (Table 3). Moreover, a positive family history for headache and migraine was found in many cases (respectively, 73.2\%, $P=0.0005$; $66 \%, P=0.02$ ) (Table 3). In the majority (95\%) of the cases reported by Yamane et al. [13] the headache started in the same year as or after the diagnosis of epilepsy; this was also confirmed in our series.

In the literature a strong association between MA and epilepsy has been suggested in both adults and children $[14,15,18]$. In adults, the MA prevalence was more elevated in cases with epilepsy (41\%) compared to those without $(25.8 \%)$ [18]. In a case-control study on children, the risk of unprovoked seizures was increased in MA (3.7-fold) but not in MO cases [14]. Differently, in our cases, MA did not increase the risk of epilepsy compared to $\mathrm{MO}$ and the prevalence of epilepsy was similar across the two groups of migraineurs (MO 5.4\%, MA 5.8\%). Moreover, in the majority of our cases (71\%) epilepsy preceded MA and this finding is not consistent with a causative role for MA in epilepsy, as previously suggested [14]. It is more likely that MA and epilepsy share a common antecedent factor.

The prevalence of epilepsy in migraineurs varies from 1 to $17 \%$, with a median of $5.9 \%[1,2]$, substantially higher
Table 3 Comparison between our cases and other series reported in the literature [13]

\begin{tabular}{|c|c|c|c|c|}
\hline \multirow{2}{*}{$\begin{array}{l}\text { Characteristics } \\
\text { of patients }\end{array}$} & \multicolumn{2}{|c|}{ Present study } & \multicolumn{2}{|c|}{ Yamane 2003} \\
\hline & $N=56$ & $\chi^{2}$ & $N=23$ & $\chi^{2}$ \\
\hline \multicolumn{5}{|l|}{ Gender } \\
\hline Female & 36 & $P=0.03$ & 13 & $P=0.376$ \\
\hline Male & 20 & & 10 & \\
\hline \multicolumn{5}{|c|}{ Age at headache onset } \\
\hline$<10$ years & 34 & $P=0.13$ & 5 & $P=0.0001$ \\
\hline$\geq 10$ years & 22 & & 18 & \\
\hline \multicolumn{5}{|l|}{ Type of epilepsy } \\
\hline Idiopathic & 21 & $P=0.13$ & 15 & $P=0.0003$ \\
\hline Cryptogenic & 32 & & 5 & \\
\hline Symptomatic & 0 & & 3 & \\
\hline \multicolumn{5}{|l|}{ Seizure type } \\
\hline Focal & 43 & $P<0.0001$ & 14 & $P=0.140$ \\
\hline Generalized & 10 & & 9 & \\
\hline \multicolumn{5}{|c|}{ Family history of headache } \\
\hline Positive & 41 & $P=0.0005$ & 12 & $P=0.546$ \\
\hline Negative & 15 & & 10 & \\
\hline n.a. & 0 & & 1 & \\
\hline \multicolumn{5}{|c|}{ Family history of migraine } \\
\hline Positive & 37 & $P=0.02$ & n.a. & n.a. \\
\hline Negative & 19 & & & \\
\hline \multicolumn{5}{|c|}{ Family history of epilepsy } \\
\hline Positive & 22 & $P=0.11$ & n.a. & n.a. \\
\hline Negative & 34 & & & \\
\hline \multicolumn{5}{|c|}{ Type of headache } \\
\hline Migraine & 46 & $P<0.0001$ & 10 & $P=0.132$ \\
\hline Tension type & 10 & & 4 & \\
\hline Not classified & 0 & & 9 & \\
\hline
\end{tabular}

Relationship between onset of headache and diagnosis of epilepsy

$\begin{array}{llll}\text { Before epilepsy } & 15 & P=0.32 & 1\end{array} \quad P=0.001$

Temporal relationship between headache and epilepsy

$$
\begin{array}{lllll}
\text { Yes } & 21 & P=0.06 & 9 & P=0.140 \\
\text { No } & 35 & & 14 &
\end{array}
$$

Type of temporal relationship between headache and epilepsy

$$
\begin{array}{lrrll}
\text { Preictal } & 2 & P=0.0002 & 2 & P=0.157 \\
\text { Postictal } & 19 & & 19 &
\end{array}
$$

n.a. not available

than the population prevalence of epilepsy which is $0.5-1 \%$ (http://www.who.int/topics/epilepsy). In a recent study on 134 children and adolescents with primary headache, the overall prevalence of epilepsy or unprovoked seizures was $4.4 \%$ [15]. Among the three cases affected by epilepsy, two had photosensitive epilepsy and one had temporal epilepsy and they all suffered from MA [15]. 
In our cases, the prevalence of idiopathic or cryptogenic epilepsy or unprovoked seizures was $3.1 \%$ in headache sufferers and $4.8 \%$ in migraineurs, similar to other reports [2-4, 15]. Moreover, in migraineurs, the risk of epilepsy was 3.2 times higher compared to subjects with ETTH, and the risk of epilepsy was not increased in MA compared to MO cases.

In children, the association between migraine and some epilepsy syndromes, such as childhood epilepsy with occipital paroxysms and benign childhood epilepsy with centrotemporal spikes reported by some authors $[2,17,19-$ 21], was not confirmed by subsequent studies [3, 22]. It has been recently reported that partial epilepsies (benign rolandic and focal cryptogenic/symptomatic epilepsy), regardless of etiology, are associated with higher rates of migraine in children [7].

Among our patients with comorbidity, focal epilepsies prevailed $(76.8 \%)$, and in migraineurs the risk of having a focal cryptogenic epilepsy was threefold higher than idiopathic epilepsy.

A PPR was found in $12.5 \%$ of our cases with comorbidity, which is more elevated than the occurrence in healthy individuals aged 6-18 (1.4\%), in patients with epilepsy $(5 \%)$ and in children with idiopathic headaches (mean $8 \%$; $8.9 \%$ in $\mathrm{MO}, 7.8 \%$ in $\mathrm{MA}, 6.7 \%$ in $\mathrm{TTH}$ ) [15, 23-26].

A positive family history of headache and migraine was found, respectively, in $73.2 \%$ and $66 \%$ of our cases. While we found no significant correlation between the positive family history of migraine and the type of headache or of epilepsy of the probands, a positive family history for epilepsy was present in $39 \%$ of our cases with comorbidity and it was more elevated compared to other series $(9.5 \%)$ [15].

In the past years, three alternative models have been proposed to explain the comorbidity between migraine and epilepsy: (a) a simple unidirectional causal explanation; (b) shared environmental risk factors; or (c) shared genetic risk factors [6]. Similar to previous studies [16, 27-29], our results are consistent with rejecting these three possibilities because epilepsy preceded migraine in the majority of our cases, and we did not find any statistical significant correlation between the positive family history of migraine and the type of headache or epilepsy of the probands.

Two rare conditions in which there is a complete overlapping between headache and epilepsy and raise problems of differential diagnosis are migralepsy and ictal headache [30-33]. In both cases, EEG recording during the headache attacks is essential for diagnosis. Migralepsy (1.5.5 ICHD-II) is a particular condition wherein a seizure occurs during or within $1 \mathrm{~h}$ after a migraine aura, and it can mime occipital lobe seizures, which might be underrecognized without EEG recording [30]. Rarely, headache (not only migraine) might represent the sole ictal epileptic manifestation, and the new term "ictal epileptic headache" was proposed to be included in both classifications [3133]. In our series nobody experienced episodes of migralepsy or ictal epileptic headache.

At present many authors believe that an increased brain excitability might increase the risk of both migraine and epilepsy and could explain the comorbidity [18, 28, 34, 35]. There is considerable interest in the possibility that genetic polymorphisms in ion channels or in genes encoding other molecules involved in the generation and the maintenance of membrane contribute to epilepsy and migraine susceptibility [35].

In conclusion, comorbidity between headache and epilepsy is common in children and adolescents. Our results confirm a strong association between migraine and epilepsy, without significant difference between MO and MA. Migraine is associated with higher rates of focal epilepsies, in particular cryptogenic epilepsies. Recurrent attacks of migraine do not seem to cause epilepsy, since in the majority of our cases, and mainly in MA, epilepsy preceded migraine. In cases with comorbidity, migraineurs do not have a major risk of post-ictal headache compared to tension-type headache sufferers. Photosensitivity on EEG and positive family history for epilepsy are frequent in children with comorbidity between headache and epilepsy.

The analysis of the clinical relationship between headache (especially migraine) and epilepsy could contribute to understanding the pathogenetic mechanisms of these two conditions, mainly when they are comorbid. Further studies, based on the present diagnostic criteria of both disorders [10-12], are needed.

Conflict of interest None.

\section{References}

1. Haut SR, Bigal ME, Lipton RB (2006) Chronic disorders with episodic manifestations: focus on epilepsy and migraine. Lancet Neurol 5:148-157

2. Andermann E, Andermann FA (1987) Migraine-epilepsy relationships: epidemiological and genetic aspects. In: Andermann F, Lugaresi E (eds) Migraine and epilepsy. Butterworth Publishers, Boston, pp 281-291

3. Andermann F, Zifkin B (1998) The benign occipital lobe epilepsies of childhood: an overview of the idiopathic syndromes and of the relationship to migraine. Epilepsia 39:9-23

4. Andermann F (2000) Migraine and the benign partial epilepsies of childhood: evidence for an association. Epileptic Disord S1:37-39

5. Ottman R, Lipton RB (1994) Comorbidity of migraine and epilepsy. Neurology 44:2105-2110

6. Ottman R, Lipton RB (1996) Is the comorbidity of epilepsy and migraine due to a shared genetic susceptibility? Neurology 47:918-924 
7. Wirrell EC, Hamiwka LD (2006) Do children with benign rolandic epilepsy have a higher prevalence of migraine than those with other partial epilepsies or nonepilepsy controls? Epilepsia 47:1674-1681

8. Thomsen LL, Kirchmann M, Bjornsson A et al (2007) The genetic spectrum of a population-based sample of familial hemiplegic migraine. Brain 130:346-356

9. Ducros A (2008) Familial and sporadic hemiplegic migraine. Rev Neurol 164:216-224

10. Headache Classification Subcommitee of the International Headache Society (2004) The international classification of headache disorders: 2nd edition. Cephalalgia 24(S1):9-160

11. International League Against Epilepsy (1989) Proposal for revised classification of epilepsies and epileptic syndromes. Commission on classification and terminology of the international league against epilepsy. Epilepsia 30:389-399

12. Engel J Jr (2006) ILAE classification of epilepsy syndromes. Epilepsy Res 70(S1):5-10

13. Yamane LE, Montenegro MA, Guerreiro MM (2004) Comorbidity headache and epilepsy in childhood. Neuropediatrics 35:99-102

14. Ludvigsson P, Hesdorffer D, Olafsson E et al (2006) Migraine with aura is a risk factor for unprovoked seizures in children. Ann Neurol 59:210-213

15. Piccinelli P, Borgatti R, Nicoli F et al (2006) Relationship between migraine and epilepsy in pediatric age. Headache 46:413-421

16. De Romanis F, Buzzi MG, Cerbo R et al (1991) Migraine and epilepsy with infantile onset and electroencephalographic findings of occipital spike-wave complexes. Headache 31:378-383

17. Clarke T, Baskurt Z, Strug LJ et al (2009) Evidence of shared genetic risk factors for migraine and rolandic epilepsy. Epilepsia 50:2428-2433

18. Leniger T, von den Driesch S, Isbruch K et al (2003) Clinical characteristics of patients with comorbidity of migraine and epilepsy. Headache 43:672-677

19. Bladin PF (1987) The association of benign rolandic epilepsy with migraine. In: Andermann F, Lugaresi E (eds) Migraine and epilepsy. Butterworth Publishers, Boston, pp 145-152

20. Giroud M, Couillault G, Arnould S et al (1989) Epilepsy with rolandic paroxysms and migraine, a non-fortuitous association. Results of a controlled study. Pediatrie 44:659-664
21. Kinast M, Lueders H, Rothner AD et al (1982) Benign focal epileptiform discharges in childhood migraine. Neurology 32:1309-1311

22. Santucci M, Giovanardi Rossi P, Ambrosetto G et al (1985) Migraine and benign epilepsy with rolandic spikes in childhood: a case control study. Dev Med Child Neurol 27:60-62

23. Kasteleijn-Nolst Trenité DGA, Hirsch E, Takahashi T (2002) Photosensitivity, visual induced seizures and epileptic syndromes. In: Roger J, Bureau M, Ch Dravet et al (eds) Epileptic syndromes in infancy, childhood and adolescence, 3rd edn. John Libbey \& Co Ltd, Eastleigh, pp 369-385

24. Kasteleijn-Nolst Trenité DGA, Silva LCB, Manreza MLG (2003) Prevalence of photo paroxysmal EEG responses in normal children and adolescents in Teofile Otoni, Brazil: 2001-2002. Epilepsia 44(S8):48

25. Wendorff J, Juchniewicz B (2005) Photosensitivity in children with idiopathic headaches. Neurol Neurochi Pol 39(S1):S9-S16

26. Piccioli M, Parisi P, Tisei P et al (2009) Ictal headache and visual sensitivity. Cephalalgia 29:194-203

27. De Simone R, Ranieri A, Marano E et al (2007) Migraine and epilepsy: clinical and pathophysiological relations. Neurol Sci 28(S2):150-155

28. Bigal ME, Lipton RB, Cohen J (2003) Epilepsy and migraine. Epilepsy Behav S2(4):13-24

29. Lipton RB, Silberstein SD (1994) Why study the comorbidity of migraine? Neurology 44(S7):S4-S5

30. Sances G, Guaschino E, Perucca P et al (2009) Migralepsy: a call for a revision of the definition. Epilepsia 50:2487-2496

31. Parisi P, Piccioli M, de Sneeuw S et al (2008) Redefining headache diagnostic criteria as epileptic manifestation? Cephalalgia 28:408-409

32. Parisi P (2009) Who's still afraid of the link between headache and epilepsy? Some reactions to and reflections on the article by Marte Helene Bjørk and co-workers. J Headache Pain 10:327329

33. Parisi $P$ (2009) Why is migraine rarely, and not usually, the sole ictal epileptic manifestation? Seizure 18:309-312

34. Yankovsky AE, Andermann F, Bernasconi A (2005) Characteristics of headache associated with intractable partial epilepsy. Epilepsia 46:1241-1245

35. Rogawski MA (2008) Common pathophysiologic mechanisms in migraine and epilepsy. Arch Neurol 65:709-714 